\title{
Relativistic plasma aperture for laser intensity enhancement
}

\author{
M. Jirka $\odot$, O. Klimo $\odot$, and M. Matys $\odot$ \\ ELI Beamlines Centre, Institute of Physics, Czech Academy of Sciences, Za Radnici 835, 25241 Dolni Brezany, Czech Republic \\ and Faculty of Nuclear Sciences and Physical Engineering, Czech Technical University in Prague, Brehova 7, 115 19 Prague, Czech Republic
}

(Received 25 May 2021; accepted 19 July 2021; published 20 August 2021)

\begin{abstract}
A substantial increase in local laser intensity is observed in the near field behind a plasma shutter. This increase is caused by the interference of the diffracted light at the relativistic plasma aperture and it is studied both analytically and using numerical simulations. This effect is only accessible in the regime of relativistically induced transparency and thus it requires a careful choice of laser and target parameters. The theoretical estimates for the maximum field strength and its spatial location as a function of target and laser parameters are provided and compared with simulation results. Our full 3D particle-in-cell simulations indicate that the laser intensity may be increased roughly by an order of magnitude, improving the feasibility of strong field QED research with the present generation of lasers.
\end{abstract}

DOI: 10.1103/PhysRevResearch.3.033175

\section{INTRODUCTION}

Nowadays, the most powerful laser systems can reach intensity on the order of $10^{22} \mathrm{~W} / \mathrm{cm}^{2}$ and the dynamics of laser-plasma interaction becomes strongly relativistic [1-3]. One example is the onset of relativistic transparency in the interaction with the overdense target [4]. The laser light cannot propagate in the overdense target, as its frequency is lower or equal to the frequency of the electron plasma oscillations. However, when the intense laser field is applied, the electron mass increases due to the relativistic motion reducing the plasma frequency. It results in a decrease of the effective target density and thus the intense part of the laser field can propagate through the plasma.

Such an effect is especially important in the interaction with a thin overdense foil, as it affects the temporal envelope of the laser pulse [5-8]. Therefore, it has an impact on ion acceleration and radiation generation $[9,10]$. It was shown that in the case of an ultraintense laser pulse, a relativistic plasma aperture is created in an ultrathin foil and the laser pulse is diffracted [11]. Since the plasma electrons are driven by the diffraction pattern of the laser field, the spatial structure of the accelerated electron beam can be controlled by varying the laser pulse parameters [11]. As the electron dynamics consequently affects the spatial-intensity distribution of the accelerated ions, such an approach could be used for optically controlled ion acceleration [12-14].

Here we study the increase of the laser field intensity caused by the interference of the light diffracted at the relativistic plasma aperture for the case of linearly polarized laser pulse. It is investigated both analytically and using $2 \mathrm{D}$ and $3 \mathrm{D}$

Published by the American Physical Society under the terms of the Creative Commons Attribution 4.0 International license. Further distribution of this work must maintain attribution to the author(s) and the published article's title, journal citation, and DOI. particle-in-cell (PIC) simulations. It is shown that due to the relativistic motion of the electrons, the laser intensity can be locally increased by almost an order of magnitude. However, this effect is only accessible in the regime of relativistically induced transparency and thus it requires a careful choice of the laser and target parameters. If the target is either under- or overdense, the relativistic plasma aperture cannot be created and the diffraction structure responsible for the field intensity enhancement is not present. The theoretical estimates for the maximum field strength and its spatial location are provided as a function of laser pulse and target parameters and compared with simulation results.

\section{INTERACTION WITH A PLASMA SHUTTER}

In the following text, we study the process of laser intensity enhancement in the interaction of an ultraintense laser pulse $\left(\sim 10^{22} \mathrm{~W} / \mathrm{cm}^{2}\right)$ with an ultrathin $(\sim 10 \mathrm{~s} \mathrm{~nm})$ plasma layer having the electron plasma density $n_{\mathrm{p}}$ greater than the value of the relativistic critical density $n_{\mathrm{c} \gamma}=\gamma n_{\mathrm{c}}$, where $\gamma$ is the relativistic Lorentz factor (of an electron having the charge $e$ and mass $\left.m_{e}\right)$ and $n_{\mathrm{c}}=\omega_{0}^{2} m_{e} /\left(4 \pi e^{2}\right)$ represents the nonrelativistic critical plasma density. The ion motion is neglected. We assume a laser pulse linearly polarized along the $y$ axis propagating in the negative $x$ direction focused to a spot of radius $w_{0}$. It has a Gaussian temporal envelope of full width at half maximum (FWHM) duration $\tau$ in the laser intensity. Its amplitude is characterized by the Lorentz invariant parameter $a_{0}=e E_{0} /\left(m_{e} \omega_{0} c\right)$, where $E_{0}$ is the amplitude of the electric field, $\omega_{0}$ is the laser angular frequency, and $c$ is the speed of light [15].

When a relativistically overdense shutter $\left(n_{\mathrm{p}}>n_{\mathrm{c} \gamma}\right)$ is assumed, the front part of the laser pulse is reflected from the foil surface. However, as the laser pulse intensity grows to its maximum value, the laser pulse starts to penetrate the target. The dominant mechanism allowing target penetration differs depending on the target thickness and density. In the case of the ultrathin foil, the electrons are pushed away due 
to the radiation pressure of the incoming laser pulse. If the thicker target is assumed, it is rather the increasing relativistic mass of electrons that causes the foil to become relativistically transparent so the rest of the laser pulse can pass through the foil. Therefore, the laser field strength at which the plasma layer becomes transparent depends on the density and thickness of the foil as well as on the laser wavelength $\lambda$. In both cases, the laser pulse acquires a steep front edge as a result of the interaction [5-7] that can be utilized, e.g., for enhanced photon emission due to the improved temporal contrast or for enhancement of ion acceleration from structured targets due to reduced development of transverse short-wavelength instabilities $[16,17]$. Here we study the interaction with the ultrathin foil. For $a_{0} \gg 1$ and an unperturbed plasma slab of thickness $l \ll \lambda$, the laser pulse strength required for penetration of the foil (meaning that the laser pulse is able to push all electrons away from the initial position of the foil by the radiation pressure) can be estimated as $[5,18,19]$

$$
a_{0}^{\max }=\frac{n_{\mathrm{p}} \pi l}{n_{\mathrm{c}} \lambda} .
$$

Naturally, the laser pulse penetrates the target near the laser axis at first. With the increase of the laser pulse strength at later times, the target becomes transparent at all points where the condition Eq. (1) for laser penetration is satisfied.

However, even for $a_{0}<a_{0}^{\max }$, the laser pulse may penetrate through the foil at least partially due to the skin effect [20]. The relativistically corrected skin depth is $l_{\text {skin }}=\sqrt{\gamma} c / \omega_{\mathrm{p}}$, where $\omega_{\mathrm{p}}=\sqrt{4 \pi n_{\mathrm{p}} e^{2} / m_{e}}$ is the plasma frequency [15]. The radiation pressure of the laser pulse will first squeeze the plasma layer of initial thickness $l$ to a thin slab of thickness $d$ while the density will grow by a factor of $l / d$. The corresponding skin depth is $c / \omega_{0} \sqrt{\gamma n_{\mathrm{c}} d /\left(n_{\mathrm{p}} l\right)}$. As shown later in the text, the amplitude of the resulting field is enhanced due to the interference of the transmitted and scattered beams. Since the strength of the scattered beam should be non-negligible to noticeably contribute to the interference, we consider the target to be transparent if the intensity of the transmitted (scattered) beam is at least of the order of $\exp (-3 / 2)$ of the incident intensity. It is a free parameter and this particular choice illustrates well the studied case $(10 \mathrm{~s} \mathrm{~nm}$ targets, intensity $10^{22} \mathrm{~W} / \mathrm{cm}^{2}$ ). If the target is very thin, the oscillating border of the plasma aperture cannot fully develop and affect the interaction. In such a case, the scattering process is not effective. For targets thicker than the skin depth, the scattered intensity is below the order of $\exp (-2)$ of the incident intensity. Therefore, in both cases, the interference enhancement is low and not interesting. Thus, assuming the intensity of the incident laser pulse is reduced by a factor of $\exp (-3 / 2)$ after passing through the squeezed plasma layer of a thickness $d$, we obtain

$$
2 k_{0} \sqrt{\frac{l d n_{\mathrm{p}}}{\gamma n_{\mathrm{c}}}}=\frac{3}{2}
$$

where $k_{0}=\omega_{0} / c$. Based on Eq. (1), the field strength $a_{0}^{\mathrm{t}}=$ $n_{\mathrm{p}} \pi(l-d) / n_{\mathrm{c}} \lambda$ is required for pushing the electrons along distance $l-d$ of the unperturbed target of density $n_{\mathrm{p}}$ to create a squeezed plasma layer. After finding the value of $d$ from Eq. (2) and inserting into $a_{0}^{\mathrm{t}}$, one may obtain the characteristic

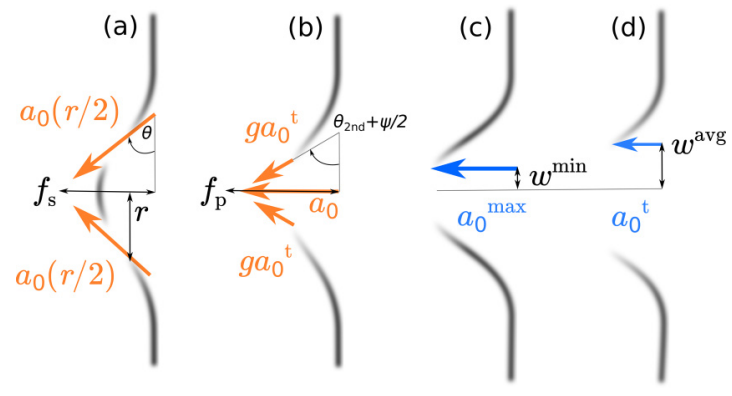

FIG. 1. Schematic of target geometry during the interaction with (a) $s$-polarized and (b) p-polarized laser pulse. (c) Minimal and (d) characteristic aperture radius for penetration of the foil.

amplitude of the field required for penetration of the target [as a penetration we consider intensity drop by a factor of $\exp (-3 / 2)]$

$$
a_{0}^{\mathrm{t}}=\frac{4 / 3 \sqrt{2} k_{0}^{2} l^{2} n_{\mathrm{p}}}{\left(3 / 4+8 / 3 \sqrt{2} k_{0} l\right) n_{\mathrm{c}}} .
$$

For the ultrathin foils with the thickness of the order of $10 \mathrm{~nm}$ assumed in this paper, $a_{0}^{\mathrm{t}} \approx 0.5 a_{0}^{\max }$. While the most intense part of the laser pulse propagates through the thin plasma slab and a relativistic aperture is formed, the electrons at the borders of the aperture oscillate in the direction of the resulting electric field, which is given by the interference of the incident laser wave with the wavefronts reflected from a relativistically overdense plasma slab [11]. In the case of $s$ polarization (the laser electric field is perpendicular to the plane of incidence), target electrons are simultaneously driven toward the laser beam axis by the ponderomotive force of the two nodes created at the target front side twice per laser period [11]. It results in an increase of the target density on the axis and therefore two separate relativistic plasma apertures are created in the vicinity of the laser beam axis at first, see Fig. 1(a) [11]. It agrees with a double lobe profile of the transmitted laser field observed in three-dimensional simulations and the corresponding asymmetry measured in the distribution of accelerated electrons and ions [11,12,21,22]. We assume that at the radial position $r$, i.e., at the outer edge of the relativistic plasma aperture, the laser pulse strength is equal to $a_{0}^{\mathrm{t}}=a_{0} \exp \left(-r^{2} / w_{0}^{2}\right)$. As the high density along the beam axis prevents the transmission of the beam close to the axis, the strength of the field passing through one aperture can be estimated as $a(r / 2)=a_{0} \exp \left[-r^{2} /\left(2 w_{0}\right)^{2}\right]$. The resulting field at the rear side of the target is created by the interference of two transmitted beams, each having the above-mentioned strength. Therefore, the amplitude of the diffracted field is approximately given by

$$
a_{0}^{\mathrm{s}}=2 a(r / 2)=2 a_{0} \exp \left[-r^{2} /\left(2 w_{0}\right)^{2}\right] .
$$

When $p$ polarization is assumed (the laser electric field is parallel to the plane of incidence), the aperture is created on the axis at first, see Fig. 1(b). Contrary to the previous case, dense electron bunches are driven alternatively from two opposite edges of the aperture toward the laser axis at the frequency $2 \omega_{0}$ [23]. The photons of the transmitted laser pulse are thus scattered by these relativistic electron bunches 
generating a number of high harmonics. The intensity of the $n$th harmonic can be approximated by a power-law $I_{n} \propto n^{-8 / 3}$ $[24,25]$. Assuming that laser field of strength $a_{0}^{\mathrm{t}}$ is scattered by the electron bunches at two opposite sides of the aperture, the interference of the generated harmonics will create a field of strength $2 g a_{0}^{\mathrm{t}}$, where $g=\sqrt{\sum_{n=2}^{N} n^{-8 / 3}}$ is the fraction of the incoming field scattered into $N$ harmonics. Due to their further interference with the field propagating along the laser axis, the resulting field amplitude can be approximated as

$$
a_{0}^{\mathrm{p}}=2 g a_{0}^{\mathrm{t}}+a_{0} \text {. }
$$

However, in both cases, the field amplification is expected to be limited by the target thickness that has to be smaller than the relativistically corrected skin depth $l_{\text {skin }}$. Since Eq. (3) is maximized at $l=l_{\text {skin }}$ for a given target density and laser wavelength, such a choice presents through Eqs. (4) and (5) the upper limit for the resulting field strength.

The longitudinal position of the maximum field strength at the rear side of the target can be estimated from the knowledge of the aperture size and propagation direction of the transmitted or scattered light, see Fig. 1. The minimal aperture radius of the foil can be estimated as

$$
w^{\min }=w_{0} \sqrt{\left|\ln \frac{a_{0}^{\max }}{a_{0}}\right|},
$$

as illustrated in Fig. 1(c).

In the case of $s$ polarization, the target surface is perturbed by the action of created electromagnetic nodes. The propagation direction $\theta$ (with respect to the laser axis) of the transmitted beam at the rear side of the target is given by the deformation of the target surface caused by radiation pressure and, therefore, it strongly depends on laser and target parameters [26,27]. However, $\tan \theta$ can be estimated as the ratio of the laser pulse strengths at the axis and at the radial distance, which are sufficiently strong to penetrate the target. Below the strength limit required for target penetration, the foil deformation is given by the distribution of intensity isosurfaces of the incoming laser beam. In such a case, the angle can be estimated as $\tan \theta=\tau c /\left(\sqrt{2 \ln 2} w_{0}\right)$. When the plasma aperture is created, the ratio of longitudinal and radial distances given by isosurfaces has to be weighted by the maximum $a_{0}^{\max }$ and the characteristic value $a_{0}^{\mathrm{t}}$ of field strengths required for penetration, which results in

$$
\tan \theta=\frac{\tau c}{\sqrt{2 \ln 2} w_{0}} \frac{w^{\min }}{w^{\text {avg }}},
$$

where

$$
w^{\mathrm{avg}}=w_{0} \sqrt{\left|\ln \frac{a_{0}^{\mathrm{t}}}{a_{0}}\right|}
$$

is considered, see Fig. 1(d). Assuming that the laser pulse starts to penetrate once $a_{0}^{\max }$ is achieved, one can expect the interference maxima to occur at the distance

$$
f_{\mathrm{s}}=w^{\min } \tan \theta
$$

from the initial target position.

In the case of $p$ polarization, the maximum field strength is created by the constructive interference of scattered har- monics with the field of the propagating laser beam. Thus, its location depends on the propagation direction of scattered photons which is a function of electron beam momentum. However, the angle of the electron bunch propagation direction evolves with time. At the beginning, the electrons are driven along the $y$ axis. At later times, the angle of propagation $\psi$ can be estimated again from the geometrical properties of the Gaussian laser pulse as

$$
\tan \psi=\frac{\tau c}{\sqrt{2 \ln 2} w_{0}} .
$$

The laser photons are scattered into a number of harmonics, each having a different angle of propagation measured with respect to the electron beam direction. Among them, the second harmonic has a dominant contribution for the interference pattern and the corresponding angle can be estimated as [28]

$$
\theta_{2 \mathrm{nd}}=\arccos \left[\frac{1+\beta \cos (\psi / 2)}{2 \beta}\right],
$$

where $\beta=\sqrt{1-1 /\left(\gamma^{\text {avg }}\right)^{2}}, \gamma^{\text {avg }}=\sqrt{1+\left(a_{0}^{\mathrm{t}}\right)^{2} / 2}$, and $\psi / 2$ is the average value of the angle at which the electron bunch propagates. Thus, the maximum field strength is expected to be achieved at the distance of

$$
f_{\mathrm{p}}=w^{\mathrm{avg}} \tan \left(\theta_{2 \mathrm{nd}}+\psi / 2\right) .
$$

\section{2D AND 3D SIMULATION RESULTS}

We compare our theoretical predictions with $2 \mathrm{D}$ and $3 \mathrm{D}$ PIC simulations performed in the code EPOCH $[29,30]$. The 2D simulation box resolved with $10125 \times 4500$ cells is spanning from $-30 \mu \mathrm{m}$ to $15 \mu \mathrm{m}$ in the $x$ direction and from $-10 \mu \mathrm{m}$ to $10 \mu \mathrm{m}$ in the $y$ direction. The laser pulse of the peak intensity $I_{0}=10^{22} \mathrm{~W} / \mathrm{cm}^{2}\left(a_{0}=69\right)$ enters the simulation box at a boundary $x=15 \mu \mathrm{m}$ and is focused to a spot of radius $w_{0}=1.5 \mu \mathrm{m}$ located at $x=0 \mu \mathrm{m}$. It has a wavelength $\lambda=805 \mathrm{~nm}$ and a Gaussian temporal envelope of FWHM duration $\tau=30 \mathrm{fs}$ in laser intensity. We performed simulations for both $s$ and $p$ polarization of the laser pulse. The $\mathrm{Al}^{13+}$ solid foil with the corresponding free electron density $450 n_{\mathrm{c}}$ and the thickness $10-60 \mathrm{~nm}$ is located at the focal spot. The initial particle temperature is set to zero.

The distributions of the transmitted laser intensity and the density of target electrons are shown in Figs. 2 and 3 for $s$ and $p$ polarization, respectively. The black contours correspond to the relativistic critical plasma density $n_{\mathrm{c} \gamma}^{\mathrm{avg}}=\gamma^{\mathrm{avg}} n_{\mathrm{c}}$. In the first figure, it is shown that during the penetration of the target having a thickness of $30 \mathrm{~nm}$, the laser field has a two-lobe structure (at $x=-1.6 \mu \mathrm{m}$ ), later creating a symmetric field distribution at $x=-2.4 \mu \mathrm{m}$. This is a significant difference from the latter case, in which an asymmetric field diffraction pattern is created due to the interference of the penetrating laser field with the field scattered by the dense electron bunches which are driven alternately from two opposite sides of the aperture (see contours of the critical density near $x=$ $-0.6 \mu \mathrm{m}, y=2 \mu \mathrm{m}$ and $x=-1 \mu \mathrm{m}, y=-2 \mu \mathrm{m}$ in Fig. 3).

The longitudinal position of the maximum field strength generated by the interference pattern at the rear side of the target is shown in Fig. 4(a). The expected values calculated from Eqs. (9) and (12) ( $x$ axis) are compared to the results 


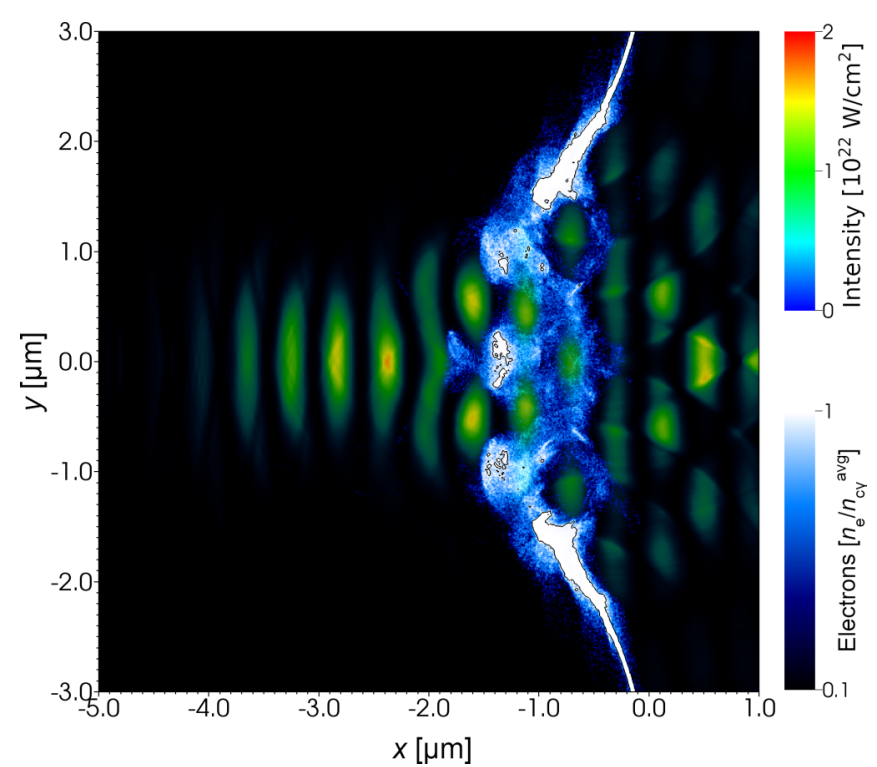

FIG. 2. The intensity of the $s$-polarized laser pulse and the density of target electrons for $I_{0}=10^{22} \mathrm{~W} / \mathrm{cm}^{2}$ and $\lambda=805 \mathrm{~nm}$. Target thickness is $30 \mathrm{~nm}$. Black contours in electron density represent the relativistic critical density $n_{\mathrm{c} \gamma}^{\mathrm{avg}}$.

obtained from PIC simulations ( $y$ axis) for different target thicknesses. The solid line is added to guide the eye for the perfect agreement between the theory and simulation results. As expected, according to the theory, the focal distance becomes shorter as the target thickness grows.

In Fig. 4(b), we compare the ratio of the first ten harmonics of the scattered light to the incident field of amplitude $E_{0}$ in the case of a $p$-polarized laser beam. The respective harmonics are obtained by filtering out all other frequency components in the Fourier transform of the laser electric field. The results

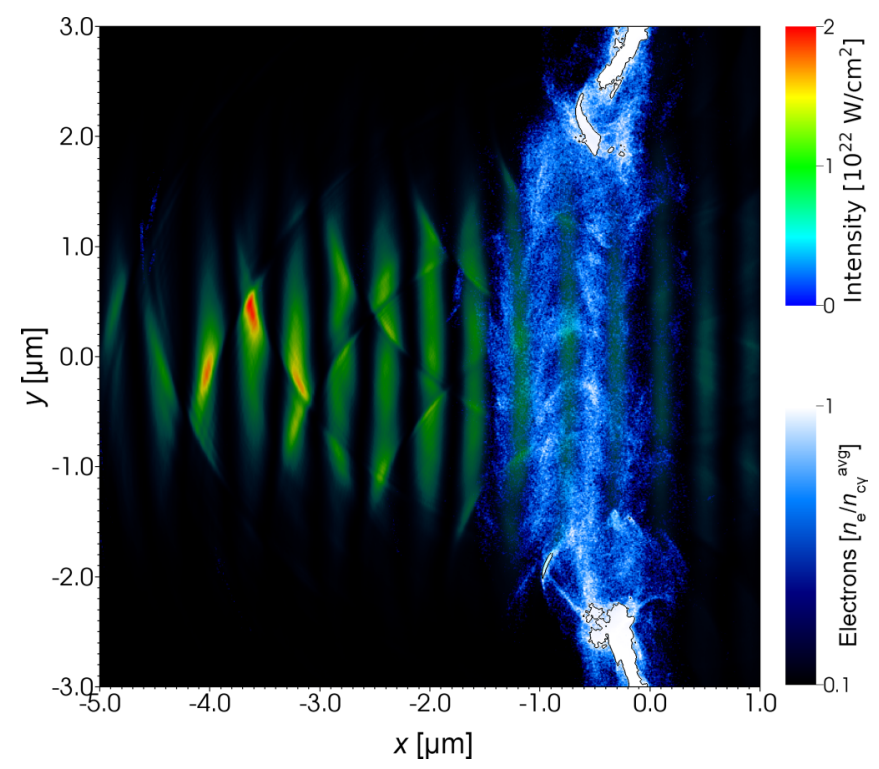

FIG. 3. The intensity of the $p$-polarized laser pulse and the density of target electrons for $I_{0}=10^{22} \mathrm{~W} / \mathrm{cm}^{2}$ and $\lambda=805 \mathrm{~nm}$. Target thickness is $30 \mathrm{~nm}$. Black contours in electron density represent the relativistic critical density $n_{\mathrm{c} \gamma}^{\text {avg }}$.
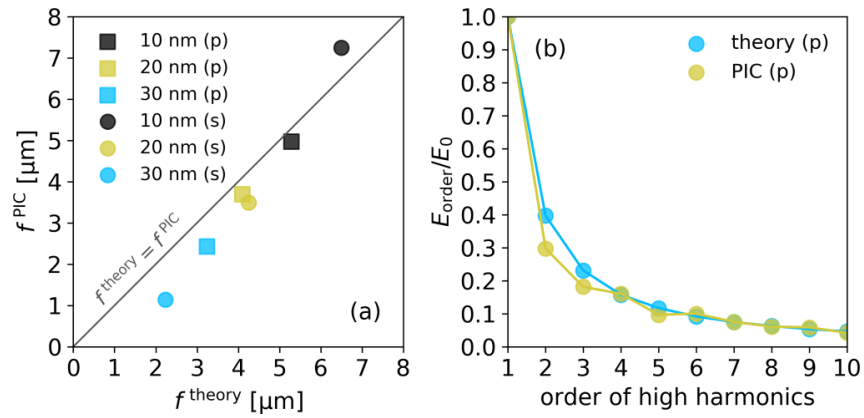

FIG. 4. (a) Longitudinal position of the maximum field strength at the rear side of the target given by Eqs. (9) and (12) and obtained from PIC simulation. (b) The ratio of the first ten harmonics of the scattered light to the incident field of strength $E_{0}$ obtained from PIC simulations and compared to the theory described in Refs. [24,25] for $p$-polarized laser beam.

obtained from the simulation with a $30 \mathrm{~nm}$ target are in good agreement with the theory described in Refs. [24,25] which was used for calculating the resulting field amplitude $a_{0}^{\mathrm{p}}$ in Eq. (5). It can be seen that the field strength for higher harmonics is small and it can be neglected. This justifies our choice of $N=10$ in Eq. (5). The electric field distributions in $k_{x}-k_{y}$ phase space ( $\mathbf{k}$ is the wave vector) for (a) $s$ and (b) $p$ polarization are shown in Fig. 5 for the same laser and target parameters. This spectrum is calculated only for the field which penetrates through the target. There is a clear evidence of high harmonics propagating at an angle in case of $p$ polarization in contrast to $s$ polarization. The $k_{x}$ spectrum is broad as the maximum intensity due to interference is very well localized, corresponding to a short spatial profile of the pulse in the longitudinal direction. The $k_{x}$ and $k_{y}$ are normalized to the vacuum wave number of the laser $k_{0}=2 \pi / \lambda$.

Figure 6 presents the comparison of the resulting field amplitude as a function of target thickness for both types of a linearly polarized laser beam. The simulation results show that the peak intensity of the diffracted field gradually grows with the target thickness as predicted by Eqs. (4) and (5). The thicker the target is, the stronger the field required for penetration, and thus the resulting diffracted field achieves higher amplitude. However, increasing the target thickness beyond

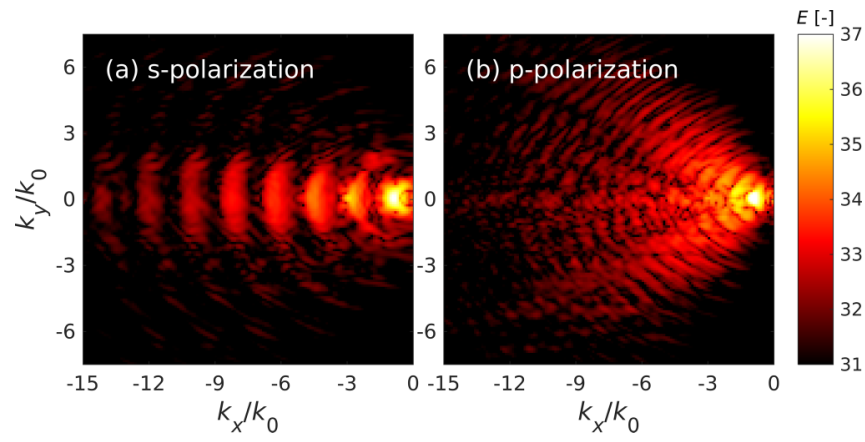

FIG. 5. The electric field intensity distribution in $k_{x}-k_{y}$ phasespace for (a) $s$ and (b) $p$ polarization at the same time step as in Figs. 2 and 3. The laser of intensity $I_{0}=10^{22} \mathrm{~W} / \mathrm{cm}^{2}$ and wavelength $\lambda=$ $805 \mathrm{~nm}$ irradiates the 30 -nm-thick target. 


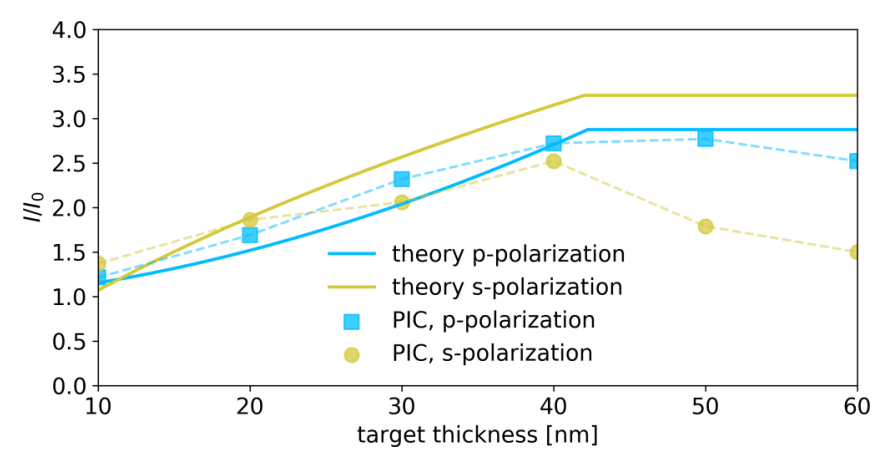

FIG. 6. Maximum intensity $I$ achieved in the interaction of the $s$ - and $p$-polarized Gaussian laser beam characterized by $I_{0}=$ $10^{22} \mathrm{~W} / \mathrm{cm}^{2}$ with the target of different thickness. Lines represent the expected values given by Eqs. (4) and (5), markers show the results obtained from PIC simulations. The relativistically corrected skin depth is $42 \mathrm{~nm}$.

the relativistically corrected skin depth $l_{\text {skin }}=42 \mathrm{~nm}$ results in more pronounced laser energy absorption in the target and thus the intensity of the diffracted field becomes reduced. The obtained results are therefore in good agreement with the theory as it has a natural limit for target thickness given by the relativistically corrected skin depth. This absorption is not taken into account in the theory and thus the theoretical curve stays constant. In the case of $p$ polarization, the first ten harmonics were considered in Eq. (5).

To check the validity of our results and get more realistic information on the field amplification due to the interference of the laser field, we carried out an additional 3D simulation for different laser and target parameters $(\lambda=1 \mu \mathrm{m}$, $\sin ^{2}$ temporal envelope, foil thickness $20 \mathrm{~nm}, n_{\mathrm{p}}=835 n_{\mathrm{c}}$ ). The simulation box of dimensions $44 \mu \mathrm{m} \times 17 \mu \mathrm{m} \times 17 \mu \mathrm{m}$ was resolved with $8800 \times 680 \times 680$ cells. The temperature of all particles was set to $5 \mathrm{keV}$. As shown in Fig. 7, the characteristic structures of the diffracted laser field in a plane perpendicular or parallel to the polarization axis (see Figs. 2 and 3) are also present in the 3D case. The horizontal and vertical slices of the laser beam correspond to $p$ and $s$ polarization from $2 \mathrm{D}$, respectively. The horizontal plane in $3 \mathrm{D}$ contains the electric field vector of the linearly polarized laser beam. The laser field amplification due to the diffraction on an evolving plasma aperture in the 3D case can be roughly estimated as $\left(a_{0}^{\mathrm{s}}+a_{0}^{\mathrm{p}}\right) / a_{0}$ (resulting in intensity enhancement by a factor of $\left.\left[\left(a_{0}^{\mathrm{s}}+a_{0}^{\mathrm{p}}\right) / a_{0}\right]^{2}\right)$, where $a_{0}^{\mathrm{s}}$ and $a_{0}^{\mathrm{p}}$ correspond to the field contributions from $s$ - and $p$-polarization components which were studied above in the 2D case, respectively. This approach predicts the maximum amplification of the laser intensity by a factor of 7.0 at a distance $3.7 \mu \mathrm{m}$ behind the shutter, which is comparable to the maximum value 7.1 obtained from 3D simulation at a distance $4.3 \mu \mathrm{m}$, see Fig. 8, having in mind that in the simulation it is not guaranteed that the field interference maxima in both planes appear simultaneously at the same position. According to the analysis of 3D simulation, the processes in $s$ and $p$ planes previously studied in the 2D case happen simultaneously and independently, while there is no dominant process responsible for intensity enhancement. Thus, this enhancement can be estimated as a combination of these two processes. The relativistic electron bunches respon-

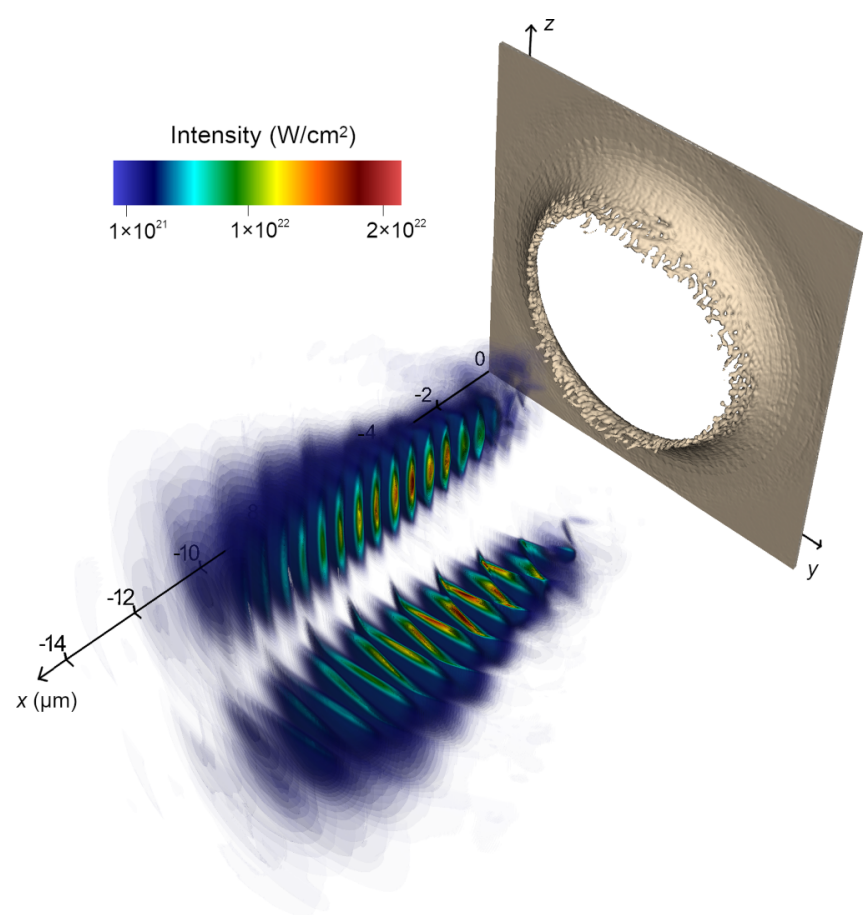

FIG. 7. The distribution of the laser intensity in horizontal and vertical slices of the laser pulse after the interaction with a foil as obtained from 3D simulation.

sible for scattering the laser field observed in the 2D case for $p$ polarization are also present in 3D simulation, see Fig. 8. We also note that in the case of long laser pulses, the ion motion should be considered in the theoretical model as it can affect the formation process of the relativistic plasma aperture and the consequent laser intensity enhancement.

So far, we have assumed an idealized laser pulse. However, the laser systems are subject to a limited contrast [31]. Due to the interaction of the laser pulse pedestal with the target, the preplasma is formed before the main laser pulse arrives and

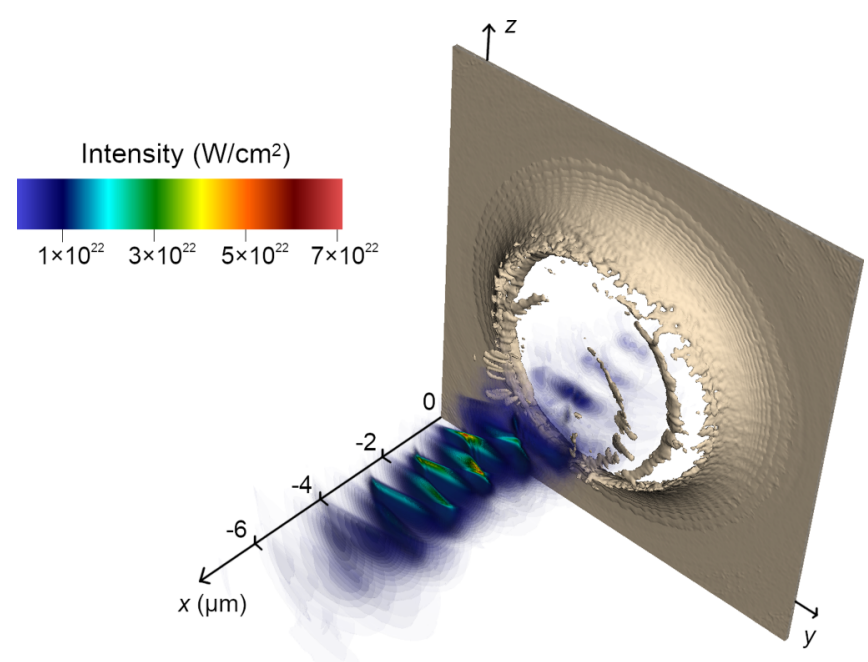

FIG. 8. The distribution of the laser intensity in the horizontal slice of the laser pulse as obtained from 3D simulation at the moment of reaching the maximum value. 
thus it can significantly affect the interaction dynamics. Considering the above-mentioned laser intensity, the pedestal of the main laser pulse can completely destroy the nm-scale foil. The foil expands to several microns along the propagation axis and therefore its density is below the relativistically corrected critical value for the main laser pulse. As a result, the main laser pulse propagates in the expanded plasma without any significant distortion or focusing. To avoid such a scenario in a real experiment, one might consider the interaction with two foils. The pedestal of the main laser pulse interacts with the first foil only. The main laser pulse then propagates through the created underdense plasma and interacts with the second foil. Such a setup allows creating a relativistic plasma aperture in the second foil, which is essential for laser pulse diffraction, resulting in the increase of the laser intensity in the near field. This setup may also provide an additional intensity increase by laser self-focusing in the pre-expanded first foil, as we partially discussed in Ref. [32]. As the prepulse is mitigated by this configuration, this feature would be especially important in the interaction with additional (nano)structured and ultrathin targets, which would be pre-expanded otherwise. The application of the single and double foil configurations in the improvement of ion acceleration from an additional target was demonstrated by simulations in Ref. [33]. As the laser intensity can be increased by almost one order of magnitude, such a setup also allows enhancing gamma radiation and pair production using the present lasers.

\section{CONCLUSION}

In conclusion, we have studied the process of laser pulse diffraction on the relativistic plasma aperture created in the interaction with an overdense ultrathin foil. Such a setup allows amplifying the laser intensity due to the interference of the transmitted and/or diffracted beam. We provide theoretical estimates for obtaining the maximum field strength and its spatial location as a function of the laser and target parameters. Using 3D numerical simulations, we have verified that the laser intensity can be increased at least seven times when proper laser and target parameters are used. This setup, based solely on relativistic plasma optics, thus presents a viable approach for obtaining localized laser intensity enhancement, which might be interesting for applications like ion acceleration, generation of gamma rays, and electron positron pair creation.

\section{ACKNOWLEDGMENTS}

Portions of this research were carried out at ELI Beamlines, a European user facility operated by the Institute of Physics of the Academy of Sciences of the Czech Republic. This work is supported by the project High Field Initiative (HIFI) CZ.02.1.01/0.0/0.0/15_003/0000449 from European Regional Development Fund (ERDF). The support of Czech Science Foundation Project No. 18-09560S is acknowledged. The support of Grant Agency of the Czech Technical University in Prague is appreciated, Grant No. SGS19/192/OHK4/3T/14. This work was supported by the Ministry of Education, Youth and Sports of the Czech Republic through the e-INFRA CZ (ID:90140). Access to CESNET storage facilities provided by the project e-INFRA CZ under the programme Projects of Large Research, Development, and Innovations Infrastructures (LM2018140), is appreciated. The simulations were performed at the cluster ECLIPSE at ELI Beamlines.
[1] S.-W. Bahk, P. Rousseau, T. A. Planchon, V. Chvykov, G. Kalintchenko, A. Maksimchuk, G. A. Mourou, and V. Yanovsky, Generation and characterization of the highest laser intensities $\left(10^{22} \mathrm{~W} / \mathrm{cm}^{2}\right)$, Opt. Lett. 29, 2837 (2004).

[2] V. Yanovsky, V. Chvykov, G. Kalinchenko, P. Rousseau, T. Planchon, T. Matsuoka, A. Maksimchuk, J. Nees, G. Cheriaux, G. Mourou, and K. Krushelnick, Ultra-high intensity-high 300TW laser at $0.1 \mathrm{~Hz}$ repetition rate, Opt. Express 16, 2109 (2008).

[3] A. S. Pirozhkov, Y. Fukuda, M. Nishiuchi, H. Kiriyama, A. Sagisaka, K. Ogura, M. Mori, M. Kishimoto, H. Sakaki, N. P. Dover, K. Kondo, N. Nakanii, K. Huang, M. Kanasaki, K. Kondo, and M. Kando, Approaching the diffractionlimited, bandwidth-limited petawatt, Opt. Express 25, 20486 (2017).

[4] J. C. Fernández, D. Cort Gautier, C. Huang, S. Palaniyappan, B. J. Albright, W. Bang, G. Dyer, A. Favalli, J. F. Hunter, J. Mendez, M. Roth, M. Swinhoe, P. A. Bradley, O. Deppert, M. Espy, K. Falk, N. Guler, C. Hamilton, B. M. Hegelich, D. Henzlova, K. D. Ianakiev, M. Iliev, R. P. Johnson, A. Kleinschmidt, A. S. Losko, E. McCary, M. Mocko, R. O. Nelson, R. Roycroft, M. A. Santiago Cordoba, V. A. Schanz, G. Schaumann, D. W. Schmidt, A. Sefkow, T. Shimada, T. N. Taddeucci, A. Tebartz, S. C. Vogel, E. Vold, G. A. Wurden, and
L. Yin, Laser-plasmas in the relativistic-transparency regime: Sci. Appl. Phys. Plasmas 24, 056702 (2017).

[5] V. A. Vshivkov, N. M. Naumova, F. Pegoraro, and S. V. Bulanov, Nonlinear electrodynamics of the interaction of ultraintense laser pulses with a thin foil, Phys. Plasmas 5, 2727 (1998).

[6] S. A. Reed, T. Matsuoka, S. Bulanov, M. Tampo, V. Chvykov, G. Kalintchenko, P. Rousseau, V. Yanovsky, R. Kodama, D. W. Litzenberg, K. Krushelnick, and A. Maksimchuk, Relativistic plasma shutter for ultraintense laser pulses, Appl. Phys. Lett. 94, 201117 (2009).

[7] S. Palaniyappan, B. Manuel Hegelich, H.-C. Wu, D. Jung, D. C. Gautier, L. Yin, B. J. Albright, R. P. Johnson, T. Shimada, S. Letzring, D. T. Offermann, J. Ren, C. Huang, R. Hörlein, B. Dromey, J. C. Fernandez, and R. C. Shah, Dynamics of relativistic transparency and optical shuttering in expanding overdense plasmas, Nat. Phys. 8, 763 (2012).

[8] W. Q. Wei, X. H. Yuan, Y. Fang, Z. Y. Ge, X. L. Ge, S. Yang, Y. F. Li, G. Q. Liao, Z. Zhang, F. Liu, M. Chen, L. Zhao, H. B. Zhuo, Y. T. Li, Z. M. Sheng, and J. Zhang, Plasma optical shutter in ultraintense laser-foil interaction, Phys. Plasmas 24, 113111 (2017).

[9] A. Henig, D. Kiefer, K. Markey, D. C. Gautier, K. A. Flippo, S. Letzring, R. P. Johnson, T. Shimada, L. Yin, B. J. Albright, 
K. J. Bowers, J. C. Fernández, S. G. Rykovanov, H.-C. Wu, M. Zepf, D. Jung, V. Kh. Liechtenstein, J. Schreiber, D. Habs, and B. M. Hegelich, Enhanced Laser-Driven Ion Acceleration in the Relativistic Transparency Regime, Phys. Rev. Lett. 103, 045002 (2009).

[10] D. Kiefer, M. Yeung, T. Dzelzainis, P. S. Foster, S. G. Rykovanov, C. L. S. Lewis, R. S. Marjoribanks, H. Ruhl, D. Habs, J. Schreiber, M. Zepf, and B. Dromey, Relativistic electron mirrors from nanoscale foils for coherent frequency upshift to the extreme ultraviolet, Nat. Commun. 4, 1763 (2013).

[11] B. Gonzalez-Izquierdo, R. J. Gray, M. King, R. J. Dance, R. Wilson, J. McCreadie, N. M. H. Butler, R. Capdessus, S. Hawkes, J. S. Green, M. Borghesi, D. Neely, and P. McKenna, Optically controlled dense current structures driven by relativistic plasma aperture-induced diffraction, Nat. Phys. 12, 505 (2016).

[12] B. Gonzalez-Izquierdo, M. King, R. J. Gray, R. Wilson, R. J. Dance, H. Powell, D. A. Maclellan, J. McCreadie, N. M. H. Butler, S. Hawkes, J. S. Green, C. D. Murphy, L. C. Stockhausen, D. C. Carroll, N. Booth, G. G. Scott, M. Borghesi, D. Neely, and P. McKenna, Towards optical polarization control of laser-driven proton acceleration in foils undergoing relativistic transparency, Nat. Commun. 7, 12891 (2016).

[13] A. Higginson, R. J. Gray, M. King, R. J. Dance, S. D. R. Williamson, N. M. H. Butler, R. Wilson, R. Capdessus, C. Armstrong, J. S. Green, S. J. Hawkes, P. Martin, W. Q. Wei, S. R. Mirfayzi, X. H. Yuan, S. Kar, M. Borghesi, R. J. Clarke, D. Neely, and P. McKenna, Near-100 MeV protons via a laserdriven transparency-enhanced hybrid acceleration scheme, Nat. Commun. 9, 724 (2018).

[14] S. D. R. Williamson, R. Wilson, M. King, M. Duff, B. Gonzalez-Izquierdo, Z. E. Davidson, A. Higginson, N. Booth, S. Hawkes, D. Neely, R. J. Gray, and P. McKenna, Selfreferencing spectral interferometric probing of the onset time of relativistic transparency in intense laser-foil interactions, Phys. Rev. Appl. 14, 034018 (2020).

[15] P. Gibbon, Short Pulse Laser Interactions with Matter (Imperial College Press, Singapore, 2005).

[16] M. Jirka, O. Klimo, Y.-J. Gu, and S. Weber, Enhanced photon emission from a double-layer target at moderate laser intensities, Sci. Rep. 10, 8887 (2020).

[17] M. Matys, K. Nishihara, M. Kecova, J. Psikal, G. Korn, and S. V. Bulanov, Laser-driven generation of collimated quasimonoenergetic proton beam using double-layer target with modulated interface, High Energy Density Phys. 36, 100844 (2020).

[18] S. S. Bulanov, C. B. Schroeder, E. Esarey, and W. P. Leemans, Optimized laser pulse profile for efficient radiation pressure acceleration of ions, Phys. Plasmas 19, 093112 (2012).

[19] S. S. Bulanov, E. Esarey, C. B. Schroeder, S. V. Bulanov, T. Zh. Esirkepov, M. Kando, F. Pegoraro, and W. P. Leemans, Radiation pressure acceleration: The factors limiting maximum attainable ion energy, Phys. Plasmas 23, 056703 (2016).

[20] E. G. Gamaliy and R. Dragila, Interaction of ultrashort laser pulses at relativistic intensities with solid targets: Relativistic skin effect, Phys. Rev. A 42, 929 (1990).
[21] L. Yin, B. J. Albright, K. J. Bowers, D. Jung, J. C. Fernández, and B. M. Hegelich, Three-Dimensional Dynamics of Breakout Afterburner Ion Acceleration using High-Contrast Short-Pulse Laser and Nanoscale Targets, Phys. Rev. Lett. 107, 045003 (2011).

[22] R. J. Gray, D. A. MacLellan, B. Gonzalez-Izquierdo, H. W. Powell, D. C. Carroll, C. D. Murphy, L. C. Stockhausen, D. R. Rusby, G. G. Scott, R. Wilson, N. Booth, D. R. Symes, S. J. Hawkes, R. Torres, M. Borghesi, D. Neely, and P. McKenna, Azimuthal asymmetry in collective electron dynamics in relativistically transparent laser-foil interactions, New J. Phys. 16, 093027 (2014).

[23] M. J. Duff, R. Wilson, M. King, B. Gonzalez-Izquierdo, A. Higginson, S. D. R. Williamson, Z. E. Davidson, R. Capdessus, N. Booth, S. Hawkes, D. Neely, R. J. Gray, and P. McKenna, High order mode structure of intense light fields generated via a laser-driven relativistic plasma aperture, Sci. Rep. 10, 105 (2020).

[24] T. Baeva, S. Gordienko, and A. Pukhov, Theory of high-order harmonic generation in relativistic laser interaction with overdense plasma, Phys. Rev. E 74, 046404 (2006).

[25] M. R. Edwards and J. M. Mikhailova, The x-ray emission effectiveness of plasma mirrors: Reexamining power-law scaling for relativistic high-order harmonic generation, Sci. Rep. 10, 5154 (2020).

[26] B. Gonzalez-Izquierdo, R. Capdessus, M. King, R. Gray, R. Wilson, R. Dance, J. McCreadie, N. Butler, S. Hawkes, J. Green, N. Booth, M. Borghesi, D. Neely, and P. McKenna, Radiation pressure-driven plasma surface dynamics in ultraintense laser pulse interactions with ultra-thin foils, Appl. Sci. 8, 336 (2018)

[27] A. McIlvenny, H. Ahmed, C. Scullion, D. Doria, L. Romagnani, P. Martin, K. Naughton, A. Sgattoni, D. R. Symes, A. Macchi, P. McKenna, M. Zepf, S. Kar, and M. Borghesi, Characteristics of ion beams generated in the interaction of ultra-short laser pulses with ultra-thin foils, Plasma Phys. Control. Fusion 62, 054001 (2020).

[28] L. D. Landau and E. M. Lifshitz, The Classical Theory of Fields, 4th ed. (Butterworth-Heinemann, Oxford, 1980).

[29] C. P. Ridgers, J. G. Kirk, R. Duclous, T. G. Blackburn, C. S. Brady, K. Bennett, T. D. Arber, and A. R. Bell, Modelling gamma-ray photon emission and pair production in highintensity laser-matter interactions, J. Comput. Phys. 260, 273 (2014).

[30] T. D. Arber, K. Bennett, C. S. Brady, A. Lawrence-Douglas, M. G. Ramsay, N. J. Sircombe, P. Gillies, R. G. Evans, H. Schmitz, A. R. Bell, and C. P. Ridgers, Contemporary particle-in-cell approach to laser-plasma modelling, Plasma Phys. Control. Fusion 57, 113001 (2015).

[31] S. Weber, S. Bechet, S. Borneis, L. Brabec, M. Bučka, E. Chacon-Golcher, M. Ciappina, M. DeMarco, A. Fajstavr, K. Falk, E.-R. Garcia, J. Grosz, Y.-J. Gu, J.-C. Hernandez, M. Holec, P. Janečka, M. Jantač, M. Jirka, H. Kadlecova, D. Khikhlukha, O. Klimo, G. Korn, D. Kramer, D. Kumar, T. Lastovička, P. Lutoslawski, L. Morejon, V. Olšovcová, M. Rajdl, O. Renner, B. Rus, S. Singh, M. Šmid, M Sokol, R. Versaci, R. Vrána, M. Vranic, J. Vyskočil, 
A. Wolf, and Q. Yu, P3: An installation for high-energy density plasma physics and ultra-high intensity laser-matter interaction at ELI-beamlines, Matter Radiat. at Extremes 2, 149 (2017).

[32] J. Nikl, M. Jirka, M. Matys, M. Kucharik, and O. Klimo, Contrast enhancement of ultra-intense laser pulses by relativistic plasma shutter, in High Power Lasers and Applications, edited by T. J. Butcher, J. Hein, P. Bakule, C. L. Haefner, G. Korn, and L. O. Silva (SPIE, 2021).

[33] M. Matys, S. V. Bulanov, M. Kecova, M. Kucharík, M. Jirka, P. Janecka, J. Psikal, J. Nikl, J. Grosz, G. Korn, and O. Klimo, Ion acceleration enhancement by laser-pulse shaping via plasma shutter, in Laser Acceleration of Electrons, edited by S. S. Bulanov, J. Schreiber, and C. B. Schroeder (SPIE, 2021). 